\title{
A Study on the Preventive Effect of Dental Caries by Chlorhexidine Mouthwash
}

By

\author{
Keiji OKADA
}

\section{Introduction}

Chlorhexidine digluconate (to be referred to as CHDG) is known for its pronounced effect by way of preventing the formation of dental plaque, as well as its curative promotion of gingival complaints.

Its administration to the human has been reported by LöE among others but, from a standpoint of caries prevention, it is necessary that a study should be undertaken in young children in whom the carious activity is especially aggressive.

In a study by FLÖRRA et al., however, there was made known some unpleasant sensation in applying CHDG to young children and, for this reason, a further study to keep this unpleasant feeling at a minimum is called for.

The present author, therefore, was concerned with a study in which the unpleasant feeling on the part of children would be kept at a minimum and, at the same time, obtaining the required caries preventive effect.

\section{Materials and Method}

An experimental group of 108 boys and girls and controls of 70 boys and girls all in the 3rd grade at the Warabi Elementary School, Saitama Prefecture, Japan, were selectively used, on whom the effect of a $0.1 \%$ aquatic solution of CHDG was tested.

Both the experimental and control groups were instructed to swallow $20 \mathrm{ml}$ of tap water and wash their mouths thoroughly by moving the buccal muscle for 5 times in succession (a simple mouthwash). Subsequently, the experimental group was given cups containing $0.1 \%$ solution of $\mathrm{CHDG}$ and, by dividing into 3 parts (one part being $20 \mathrm{ml}$ ), they were told to rinse their mouths at three intervals with 10 seconds devoted to one rinse, washing their mouths with tap water at the end of an experiment. This mouthwash was performed for 212 times altogether through a school year. The controls, on the other hand, were instructed to gargle with tap water alone for the same length of time. This was carried out after school lunch.

In conducting this experiment, questions were asked of children in the experimental group to learn if they experienced any unpleasant feeling everyday in the first month and, later, on a weekly basis. Their response to the question remained negative.

Three regular examinations, at the commencement, after 6 months and one year of the present experiment, were made of mesial, distal, labial and lingual surfaces of 
Table 1 A comparison of new dental caries between the first and second as well as the first and third examinations

\begin{tabular}{|c|c|c|c|c|c|}
\hline & & $\begin{array}{c}\text { Experimental } \\
\text { group }(n=103)\end{array}$ & Controls $(n=70)$ & Difference & Value \\
\hline \multirow{2}{*}{ 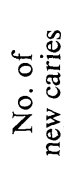 } & $\begin{array}{l}\text { Difference between } 1 \text { st } \\
\text { and } 2 \text { nd. }\end{array}$ & $\begin{array}{c}M \pm S D \\
0.333 \pm 0.707\end{array}$ & $\begin{array}{c}M \pm S D \\
0.586 \pm 0.978\end{array}$ & 0.253 & 2. $008^{*}$ \\
\hline & $\begin{array}{l}\text { Difference between 1st } \\
\text { and 3rd. }\end{array}$ & $0.593 \pm 0.912$ & $1.057 \pm 1.286$ & 0.464 & 2. $829 * *$ \\
\hline \multirow{2}{*}{ 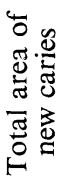 } & $\begin{array}{l}\text { Difference between 1st } \\
\text { and } 2 \text { nd. }\end{array}$ & $0.407 \pm 0.972$ & $0.614 \pm 1.045$ & 0.207 & 1. 371 \\
\hline & $\begin{array}{l}\text { Difference between } 1 \text { st } \\
\text { and 3rd. }\end{array}$ & $0.713 \pm 1.247$ & $1.086 \pm 1.247$ & 0.373 & 1.933 \\
\hline
\end{tabular}

the maxillary and mandibular central and lateral incisors, occlusal, mesiodistal and buccolingual surfaces of the maxillary and mandibular first molars $(9$ dental surfaces altogether) in an effort to establish evidence of new cariogenesis during the specific periods.

\section{Findings}

\subsection{The number of new carious surface per individual}

When the number of new carious teeth and carious surfaces were compared between the experimental and control groups at the first (commencement of the study) and second examinations (after 6 months), the rate of caries incidence was less in the experimental group at a risk of $5 \%$. When this was compared with the third examination (at the completion of study after one year), the rate of caries inhibition was significant at a risk level of $1 \%$.

\subsection{The rate of new caries incidence}

As given in Table 1, there was observed no statistical difference between the first and second examinations nor between the first and third examinations regarding the rate of new caries incidence (Table 1 ).

\subsection{Caries surfaces on different tooth segments}

Although there was no statistical difference between the first and second examinations nor between the first and third examinations regarding carious surfaces on different tooth segments, the incidence of new caries was observed mostly on the occlusal surface of mandibular first molars in common with the experimental and control groups accounting for as much as $80 \%$ of all the new caries incidences.

Next in order, new caries was found on the maxillary lingual and mandibular lingual and mandibular buccal planes as well as on the mesiodistal and labial planes of maxillary anteriors. As for the mandibular anteriors, only 2 cases of new caries were attested to (Table 2).

\subsection{The number of new carious surfaces per individuals}

Between the 1st and 2nd examinations, a statistical difference at a risk level of 
Table 2 A comparison of new caries area surface between 1st and $3 \mathrm{rd}$ examinations

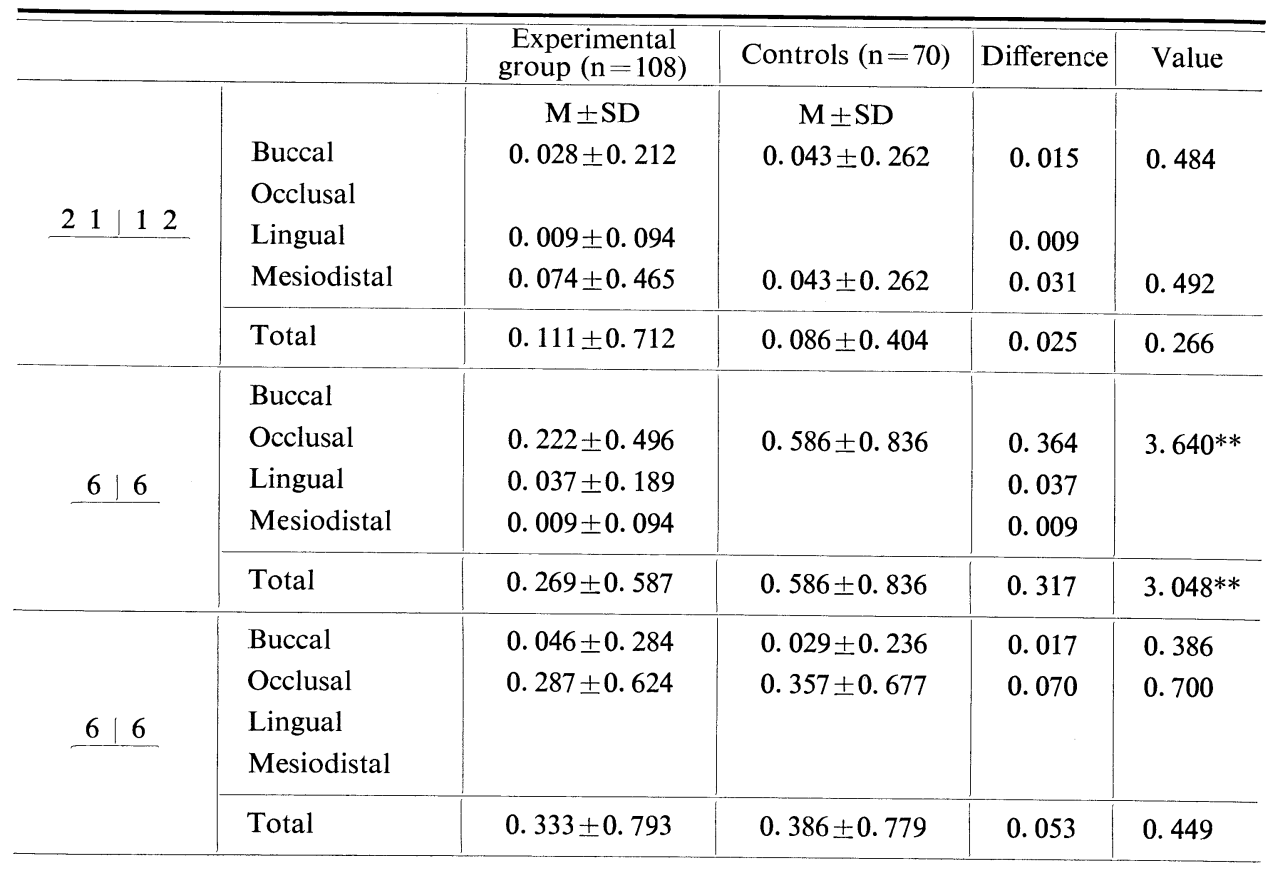

Table 3 Caries inhibition rates between 1 st and 2 nd as well as 1 st and 3 rd

\begin{tabular}{c|lcc|ccc}
\hline \multirow{2}{*}{} & \multicolumn{2}{c|}{ Difference between 1st and 2nd } & \multicolumn{3}{c}{ Difference between 1st and 3rd } \\
\cline { 2 - 6 } & \multicolumn{1}{c}{$\hat{\delta}$} & \multicolumn{1}{c|}{ Total } & \multicolumn{1}{c}{ 今 } & \multicolumn{1}{c}{ 早 } & \multicolumn{1}{c}{ Total } \\
\hline A & $30.19 \%$ & $28.53 \%$ & $29.30 \%$ & $39.05 \%$ & $21.07 \%$ & $39.78 \%$ \\
B & 32.50 & 51.57 & 43.17 & 47.20 & 40.34 & 43.90 \\
C & 26.00 & 48.28 & 38.46 & 42.16 & 36.13 & 39.43 \\
D & 17.44 & 47.57 & 33.71 & 34.40 & 34.42 & 34.35
\end{tabular}

$5 \%$ was calculated for the occlusal surfaces of right and left maxillary and mandibular first molars, whereas between the 1st and 3rd examinations a statistical difference for the same occlusal surfaces was at $1 \%$ risk level. When the carious surfaces of new incidence were compared between the experimental and control groups, there was no definite statistical difference at an interval of 6 months. However, after one year a statistical difference for the boys and girls combined was found at $5 \%$ risk level.

3.5 The rate of caries inhibition

The rate of caries inhibition by an application of CHDG was calculated between the first and second as well as the first and third examinations. As compared with the control group, the experimental group gave a total rate of caries inhibition from 30 to $40 \%$ (Table 3 ). 


\subsection{Influence on the color deposition and oral soft tissue}

After the commencement of the present study, a weekly oral examination was conducted for the first one month as to the color deposition on the teeth, and presence of any inflammatory lesion or exfoliation of oral soft tissue. Throughout the entire experimental period, there were found several cases in whom a certain degree of the color deposition was observed. This color deposition, however, disappeared after two days' rest interval at the end of every week.

\section{Discussion}

Bis-(p-chlorophenyl diguanide)-hexane is usually used in the form of alcohol soluble Chlorhexidine digluconate and is commercially sold under the name of Hibitane.

Following a report by FitzGerald on the caries prevention of CHDG in experimental animals in 1968, many studies have been published to date including LöE and all of these investigators are agreed as to its pronounced effect on inhibiting the formation of dental plaque and curative promotion of gingival complaints. For example, LöE and others confirmed a definite inhibition of plaque formation by the coating of $2.0 \%$ CHDG on dental surfaces or a mouthwash prepared from $0.2 \%$ aquatic solution and GUERMO among others reported on the same effect by a dentifrice containing CHDG. Thus, there is a fair body of the published literature on CHDG in experimental animals, in vitro and actual clinical applications. In Japan, ARITA, YAMADA and others have published their findings to the same effect.

From these previous data, it is reasonable to suppose that chlorhexidine digluconate would greatly contribute to the prevention of caries. But, at the same time, it is necessary that its effect on the occlusal surface in young children should be sufficiently confirmed before it could be applied to them on a large scale.

In this belief, the author carried out the present study directed to the third grade elementary school children of both sexes. As is known from the above-mentioned tables, the effect of CHDG in preventing dental caries by a mouthwash of $0.1 \%$ solution was confirmed to be satisfactory. Much of this effect of caries prevention, it is thought, should be ascribed to the inhibition of plaque formation by CHDG. FLÖRRA in his study with CHDG mouthwash reported cases in whom the color deposition occurred on the dental surface or silicate fillings additionally to several others in whom the exfoliation of soft tissue and complaints of pains were observed and concluded a solution of $0.1 \%$ would be most suitable from a standpoint of the least unpleasantness.

The author chose this particular concentration on the basis of the previous reports supplemented by his own data.

Prior to the commencement of this experiment, subjects were instructed to do their mouthwashing in a satisfactory manner and rinse their mouths sufficiently after CHDG mouthwash by use of tap water by inclining their bodies in a forward position. These instructions which were carefully carried out are thought to have eliminated the unpleasant factors in an application of CHDG.

Throughout one year over the present experiment, careful oral examinations established the fact that inflammations of the oral mucosa, tongue or gingiva, ex- 
foliation of other soft tissue and pains were absent altogether. However, there were some cases in whom the deposition of a yellowish brown color was found but it did not interfere with the external appearance of subjects and the color disappeared after two days' rest interval at the end of a week. It may be mentioned in passing that, since there was no subject who had some kind of a silicate filling, the discoloration of a silicate filling remained unexplored.

Despite the fact that the inhibition of the formation of dental plaque by chlorhexidine digluconate is widely recognized, its actual clinical application at present seems to be limited even if much effect can be expected in caries prevention. The reason for this may be found in possible unpleasant sensation; therefore, if sufficient attention is paid to its concentration, quantity, method and subsequent control satisfactorily, much effect will be brought about in the prevention of dental caries through an application of CHDG.

\section{Conclusions}

As a result of the study, the author arrived at the conclusions as follows.

1. A comparison of two groups of third grade elementary school children, the experimental group using a $0.1 \%$ solution of chlorhexidine digluconate as a mouthwash for one year (108 pupils) and a control group (70 pupils) rinsing their mouths with tap water, revealed that, as compared with controls, the experimental group registered a less number of new caries at a risk level of $5 \%$ at the end of 6 months and, at the end of a year, a statistical difference between the two groups was at a risk level of $1 \%$.

2. The number of new caries on the maxillary first molars was less in the experimental group at a risk level of $1 \%$.

In terms of different dental surfaces, the incidence of new caries on the occlusal surface of maxillary first molars was less in the experimental group at a risk level of $5 \%$ after 6 months and at $1 \%$ after a year.

3. These findings are to be ascribed to the effect of CHDG and its rate of caries inhibition within the scope of the present study is anywhere from 30 to $40 \%$.

4. Throughout one year observation period, there were seen some cases in whom the color deposition took place, though it was difficult to detect them unless carefully observed. However, this type of discoloration disappeared after two days interval at the end of every week. There were no other subjective unpleasant complaints on the part of subjects under study.

A clinical application of chlorhexidine digluconate especially with the minimum of unpleasant complaints will form one of the important problems in connection with early prevention of dental caries. 